\title{
BIOSYNTHESIS OF GOLD NANOPARTICLES BY BIOSORPTION USING NEOSARTORYA UDAGAWAE: CHARACTERIZATION AND INVITRO EVALUATION
}

\author{
V. JHANSI LAKSHMI ${ }^{*}$, K. P. KANNAN ${ }^{2}$ \\ 1Department of Biotechnology, Anna University, Chennai 641047, Tamil Nadu, India, ${ }^{2}$ Department of Biotechnology, Bannari Amman \\ Institute of Technology, Sathyamangalam 638401, Tamil Nadu, India \\ Email: jhansi.minnu@gmail.com
}

Received: 24 Jun 2016 Revised and Accepted: 09 Sep 2016

\begin{abstract}
Objective: The present study was aimed to investigate gold nanoparticles synthesized by fungal isolate Neosartorya udagawae and determination of their stability in biofluids to probe their aptness in drug delivery applications.

Methods: In this procedure, gold nanoparticles were prepared by biosynthesis using seven days old culture of Neosartorya udagawae and aqueous chloroauric acid. After the complete reaction, the fungal biomass was subjected to UV-Vis, XRD, FT-IR Spectrum analysis, TEM, Zeta potential, SEM and EDX analysis.

Results: Intra/extracellular synthesis of gold nanoparticles was confirmed by a sharp peak at $526 \mathrm{~nm}$ in UV spectroscopy. SEM, TEM analysis demonstrates the spherical shape of AuNPs with an average diameter of $50 \mathrm{~nm}$ and XRD confirm the crystalline gold nanoparticles. FTIR analysis reveals the presence of the protein shell around the gold nanoparticles. The zeta potential value of AuNPs was- $36 \mathrm{mV}$ which confirmed the stability of nanoparticles dispersion. Gold nanoparticles have shown high stability in biofluids of Bovine Serum Albumin and Phosphate Buffer Saline at pH-5, pH-7and pH-9 which mimic the human colonic biological environment.
\end{abstract}

Conclusion: The fungal synthesis of AuNPs has been experimentally demonstrated and their stability in BSA, 10\% NaCl and PBS at pH-7. This might be a promising option for drug delivery applications in carcinogenic colon disorders in human beings.

Keywords: Gold nanoparticles (AuNPs), Neosartorya udagawae (NU), Biological synthesis, stability, Czapex Dox Agar (CDA), Phosphate Buffer Saline (PBS), Bovine Serum Albumin (BSA)

(C) 2016 The Authors. Published by Innovare Academic Sciences Pvt Ltd. This is an open access article under the CC BY license (http://creativecommons. org/licenses/by/4. 0/) DOI: http://dx.doi.org/10.22159/ijpps.2016v8i11.13665

\section{INTRODUCTION}

Biological synthesis of nanoparticles is unloading amplified attention in the recent past by the emerging intersection between nanotechnology and biotechnology. Nano-biotechnology is an imminent field in the nanoscience. It is the control of biological material to yield nanocomposites of various sizes and shapes. Nanosized particles up to the range of $100 \mathrm{~nm}$ exhibit dynamic properties like distribution, morphology, surface area [1] and exhibit unusual optical, chemical, photoelectrochemical, electronic properties [2] and are promising carriers in drug delivery applications [3]. In earlier days nanoparticles were produced by mechanical and chemical methods employing toxic chemical reducers, as a result nanoparticles produced were not suitable for biomedical applications. To avoid this, researchers focused their interest on non-toxic and environmentally friendly nanoparticles by microorganisms like fungi, [4] bacteria, [5] and algae [6] for the synthesis of nanoparticles which offers numerous benefits with compatibility in biomedical applications. Among all the reported microorganisms, Mycological synthesis is most suitable for the reduction of gold because of ease handling, fast downstream processing and feasibility of large scale nanoparticles synthesis. The intracellular synthesis of nanoparticles is a two-step process, the first is trapping metal ions on the fungal cell surface through electrostatic interaction of positively charged groups in enzymes present in the cell wall mycelia, secondly to metal ions reduced within the cell wall which increase the synthesis of nanoparticles [7]. Gold nanoparticles have been used in various applications for their high electrical conductivity, high surface area, easy functionalization, high stability and corrosion resistance, targeted delivery in cancer treatment, gene therapy, biosensors and magnetic resonance imaging [8]. Epicoccum nigrum fungi isolated from gold mine soil in Iran [9] and alkalotolerant actinomycetes Rhodococcus species [10] were good biological tools for AuNPs synthesis. Based on the above literature, the present study aimed to synthesize intracellular synthesis of gold nanoparticles from fungus Neosartorya udagawae isolated from Indian Kolar gold mine soil. Biosynthesised AuNPs were characterized by UV-Spec, SEM, TEM, and zeta potential, EDX, XRD and FT-IR.

\section{MATERIALS AND METHODS}

\section{Chemicals}

Czapex Dox Agar (CDA), Czapex Dox Broth (CDB), Gold (III) chloride trihydrate salt $\left(\mathrm{HAucl}_{4}\right)$, Chloramphenicol antibacterial agent, Whatman filters paper procured from Hi-media.

\section{Isolation of fungal Sp. Neosartorya udagawae}

Neosartorya udagawae fungal Sp. isolated from Kolar gold mine $\left(12.961736^{\circ} \mathrm{N}\right.$ and $\left.78.2700721^{\circ} \mathrm{E}\right)$ soil and the isolate was frequently subcultured on the CDA (fig. 1a). Seven days old culture was inoculated into $100 \mathrm{ml}$ of CDB broth. Briefly, dissolving $3.5 \mathrm{~g}$ of CDB in deionized water and sterilized at $15 \mathrm{psi}$ pressure $\left(121^{\circ} \mathrm{C}\right)$ for $15 \mathrm{~min}$. Chloramphenicol was added to avoid contamination. Subsequently, the media were cooled to room temperature and incubated on a rotary shaker at $35^{\circ} \mathrm{C}$ with $120 \mathrm{RPM}$ for seven days and maintained at $\mathrm{pH} 3$. Then the culture was filtered through Whatman filter paper and washed thrice with deionized water to remove media components. The filtered fungal biomass was used for experimental studies [11]

\section{Biosynthesis of gold nanoparticles}

One gram of Neosartorya udagawae fungal biomass was added to each flask containing $100 \mathrm{ml}$ different concentrations of chloroauric acid $\left(\mathrm{HAuCl}_{4}\right)$ viz., $2 \mathrm{mmol}, 4 \mathrm{mmol}, 6 \mathrm{mmol}, 8 \mathrm{mmol}$ and $10 \mathrm{mmol}$. The mixture was incubated for $24 \mathrm{~h}$ on a rotary shaker with 120 $\mathrm{RPM}$ at $35{ }^{\circ} \mathrm{C}$ to complete the reaction. The positive and negative control were maintained in the same condition. The synthesized AuNPs were characterized by the color change of the solution and confirmed by ultraviolet-visible spectroscopy. AuNPs were isolated 
and concentrated by repeated centrifugation at 8000 RPM for 10 min. Then, the supernatant was displaced by distilled $\mathrm{H}_{2} \mathrm{O}$ and subjected to UV-Visible Spectroscopy. After the formation of AuNPs in fungal biomass, it was collected by centrifugation at 8000 RPM for $10 \mathrm{~min}$. The supernatant was discarded and dried in hot air oven for $24 \mathrm{~h}$ at $60^{\circ} \mathrm{C}$. Finally, the purified powder was collected and used in the SEM, TEM, Zeta potential, EDX, XRD and FTIR analysis [11].

\section{Characterization of nanoparticles}

\section{UV-Visible spectrophotometry analysis}

The characterization of gold nanoparticles was carried out by UVVis spectrophotometer (Perkin-Elmer Lamda-45) and measurements (200 nm to $700 \mathrm{~nm}$ ) were taken at a resolution of 1 $\mathrm{nm}$ using $1 \mathrm{~cm}$ path quartz cuvette by Specrod 210 plus-223F1427.

\section{SEM and TEM analysis}

TEM analysis of synthesized gold nanoparticles was carried out using the Technai Sprit HT: 120KV Electron Source: LaB6. Micrographs were obtained using a JEOL (6360) JED-2300 analysis station operating at 200 KV. (Model: Qunata 250 Detector: Everhart-Thornley Detector Electron Source: Tungsten) to visualize the particle size, distribution and concentration of nanoparticles. A thin layer of the sample was prepared on a carbon coated copper grid by dropping a very small amount of the sample on the grid and the extra powder was removed using blotting paper and the SEM grid was allowed to dry under a mercury lamp for $5 \mathrm{~min}$. The images were captured in SEM mode at the desired magnification of $16000 \mathrm{x}$ and $30000 \mathrm{x}$ at $200 \mathrm{KV}$.

\section{Energy dispersive X-ray spectrometry analysis}

The structural features of the produced gold nanoparticles were examined by SEM (QUANTA, 200) equipped with energy dispersive X-ray spectrometry (EDS). The EDS analysis evaluates the amount of AuNPs present in the biomass. The samples were prepared in the same way as described for SEM analysis.

\section{Zeta potential analysis}

Zeta potential of the sample was measured by Photon Correlation Spectroscopy (Zeta Sizer 3000 HAS, Malvern, UK). Samples were diluted appropriately with the aqueous phase of the formulation to get optimum kilo counts per second (Kcps) of 50-200 for measurements and $\mathrm{pH}$ of diluted samples ranged from 6.9-7.2. Zeta potential measurements were carried out at $25{ }^{\circ} \mathrm{C}$ and the electric field strength was around $23.2 \mathrm{v} / \mathrm{cm}$.

\section{$\mathrm{X}$-ray diffraction analysis}

XRD measurements of the intracellular biosynthesized AuNPs were done by coating the dried nanoparticles on the XRD grid. The spectrum was recorded using Panalytical Xpert Pro, the Netherlands on a Phillips PW 1830 operating at a voltage of $40 \mathrm{KV}$ and current of $20 \mathrm{~mA}$ with CuK radiation.

\section{FTIR analysis}

FTIR spectroscopy measurements were carried out to identify the possible functional groups responsible for the reduction of gold ions to gold nanoparticles and the biomolecules present on the gold surface. The Fourier transforms infrared (FTIR) spectra of the synthesized gold nanoparticles recorded using JASCO FT/IR-4100 type a spectrometer with a $4 \mathrm{~cm}^{-1}$ resolution. This investigation was carried out within the range of 4000 to 400 wave number $\mathrm{cm}^{-1}$

\section{Viability test of Neosartorya udagawae}

A lapful of inoculum from the reaction flask exposed to $\mathrm{HAuCl}_{4}$ solution by streaking onto the $\mathrm{CDA}$ plate at $\mathrm{pH} 3$. In addition, the untreated Neosartorya udagawae was also streaked on CDA supplemented with $1 \mathrm{mmol}$ to $10 \mathrm{mmol}$ concentration of $\mathrm{HAuCl}_{4}$. All the plates were observed for growth after incubation at $28^{\circ} \mathrm{C}$ for $5-7$ $\mathrm{d}[12]$.

\section{In vitro stability of gold nanoparticles}

In vitro stability of Fungal-AuNPs was tested in the presence of $\mathrm{NaCl}$ BSA, and Phosphate Buffer Saline of pH5, pH7 and pH9 solutions. 1 $\mathrm{ml}$ of AuNPs solution was added to small screw-capped bottles containing $1 \mathrm{ml}$ of each $10 \% \mathrm{NaCl}, 0.5 \% \mathrm{BSA}$, PBS pH-5, pH-7 and pH-9 solutions respectively and incubated for $24 \mathrm{~h}$. The stability of gold nanoparticles with respect to SPR band was determined by recording UV-Visible spectroscopy after $24 \mathrm{~h}[13,14]$.

\section{RESULTS AND DISCUSSION}

Seven days old fungus isolated on CDA from Indian Kolar gold field mine soil (fig. 1a) was inoculated in $\mathrm{CDB}$ at pH3 and incubated for seven days at $120 \mathrm{RPM}$ at $35^{\circ} \mathrm{C}$ on a rotary shaker (fig. 1b). Among various concentrations between $2 \mathrm{mmol}$ to $10 \mathrm{mmol}$ of $\mathrm{HAuCl}_{4}$ intraextracellular synthesis of AuNPs occurred only in $2 \mathrm{mmol}$ with $1 \mathrm{~g}$ Neosartorya udagawae. Fungal biomass changed from yellow to light pink on $12 \mathrm{~h}$ incubation as shown in fig. $1 \mathrm{c}$ is in agreement with the extra-/intracellular biosynthesis of gold nanoparticles by the fungus Penicillium Sp. [15]. Nearly, 90-95\% of conversion of gold ions to nanoparticles was achieved within $24 \mathrm{~h}$ through a complete change to dark purple color of biomass (fig. 1d) which is a visual indication of intracellular accumulation of gold nanoparticles. Bio-reduction is the foremost mechanism in metallic nanoparticles synthesis [16]. Surfactants like acetyl trimethyl ammonium bromide, sodium dodecyl sulfate and sodium citrate are used for stabilization of nanoparticles commercially [17]. Enzymes such as NADH and nitrate-dependent reductases of Fusarium oxysporum and Aspergillus flavus, act as capping agents naturally [18]. Proteins, carbohydrates, enzymes and other molecules present in the cell membrane and cytoplasm of the fungal biomass of Neosartorya udagawae are responsible for stability and capping of AuNPs [19]. Earlier studies confirm the formation of AuNPs in intra and extra cellular reduction of $\mathrm{Au}(\mathrm{III})$ to $\mathrm{Au}(0)$ under similar conditions by the change of biomass color to purple [20-24]. The color of the solution is directly proportional to the concentration of biomass and aqueous gold solution. After one day incubation, no significant color change was observed it indicate the completion of reduction. Positive and negative control also remained in the same condition as there was no color change in the medium. Hence synthesized AuNPs reaction mixture was used for the characterization.
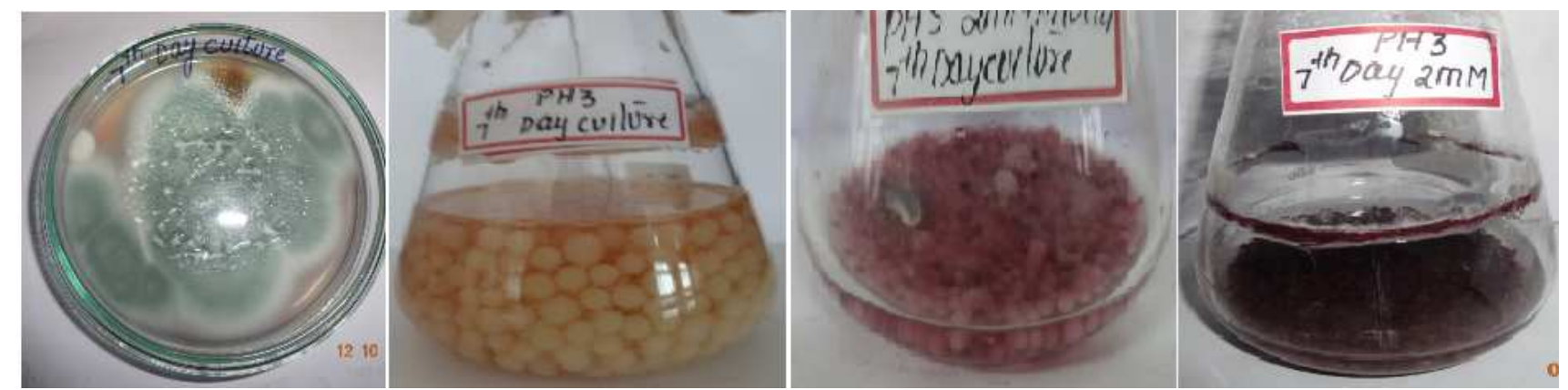

Fig. 1: Seven days old culture of $N$. udagawae on (a) CDA (b) CDB (c) Color development as a plasmon resonance in N. udagawae biomass mediated synthesis of AuNPs in 2 mmol HAuCl 4 aqueous solution in $12 \mathrm{~h}$ (d) Purple color after $24 \mathrm{~h}$ 


\section{UV-Visible spectroscopy}

The absorption maxima observed at $526 \mathrm{~nm}$ due to the excitation of surface plasmon resonance in UV corresponding to the presence of AuNPs which indicates the nano status and stability of the gold nanoparticles. There is no time-dependent change in the UV-Vis absorption spectra noticed in curve (fig. $2 \mathrm{~b}$ ) clearly indicating that the AuNPs in the aqueous phase are extremely stable with no precipitation. The enzymes secreted by fungal biomass may play a vital role in the reduction of aqueous AuCl4-ions and in the present case single band indicate the spherical shape of gold nanoparticles.

The UV-Vis absorption maxima were analogous to the gold nanoparticles synthesized by Spirulina subsalsa [25].
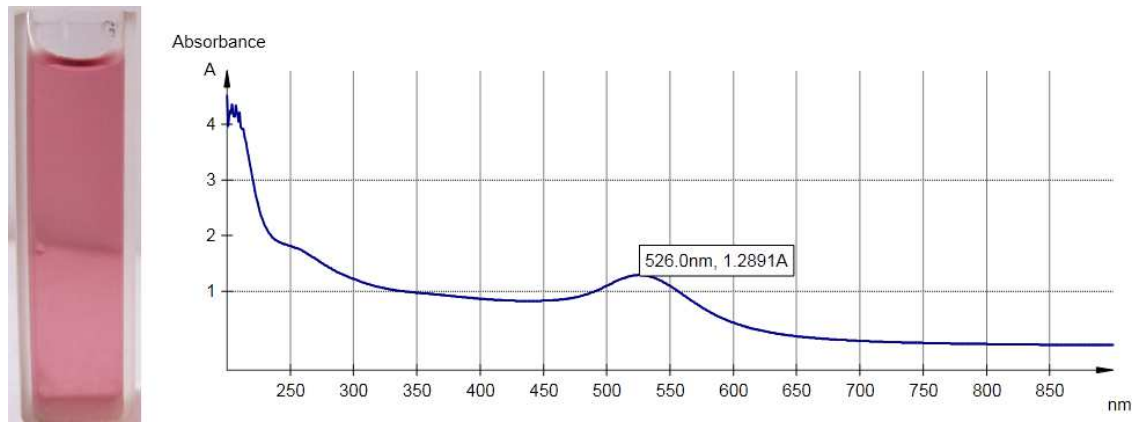

Fig. 2: (a) Pure gold nanoparticles after centrifugation (b) strong SPR band absorption peak at about $526 \mathrm{~nm}$ in UV

\section{TEM and SEM analysis}

The presence of spherical shaped AuNPs in different regions in which discrete AuNPs can clearly be discerned in average diameter of $50 \mathrm{~nm}$ in TEM images (fig. 3). The AuNPs sizes were analogous to the Gold nanoparticles of Penicillium chrysogenum [26]. The SEM images of AuNPs captured in $4 \mu \mathrm{m}$ and $5 \mu \mathrm{m}$ respectively (fig. 4) Shown bioabsorbed spherical shaped gold nanoparticles with $50 \mathrm{~nm}$ in size by glittering spots on the cell wall surfaces of $N U$ confirmed the TEM results. This trapping may be followed by an enzymatic reduction of the metal ions, leading to their aggregation and formation of nanoparticles. The SEM images were analogous to the results obtained earlier in the case of AuNPs from Arthrobacter globiformis151B [27]. Both the SEM and TEM results support the UV-Vis single band spectra which is evidence for the spherical shaped gold nanoparticles.

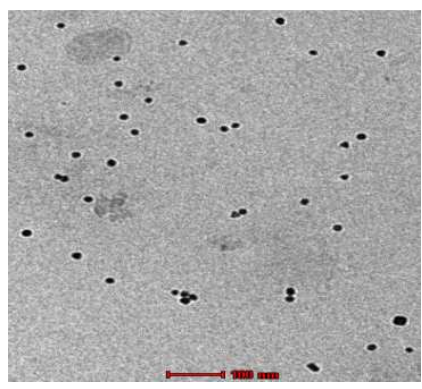

Fig. 3: TEM image of biosynthesized AuNPs

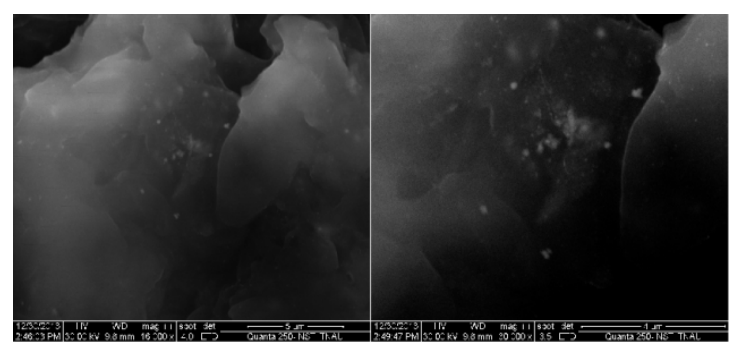

Fig. 4a: SEM images of the biosynthesized AuNPs

\section{Energy dispersive $x$-ray}

The energy dispersive X-ray analysis recorded in the spot profile mode from one of the densely populated AuNPs regions on the surface of the film gave a peak at $2.00 \mathrm{KeV}$ and this signified the presence of gold [15]. Strong signals from C, $\mathrm{O}$ and weak signals from $\mathrm{Au}, \mathrm{Cl}$ and $\mathrm{K}$ atoms were observed. The carbon and oxygen peaks ascribed to the biomolecules present in Neosartorya udagawae. The $\mathrm{Cl}$ signal indicates the presence of small amounts of AuCl4-ions in the investigated region and signals are likely due to Xray emission from enzymatic proteins present in the cell wall of fungal biomass (fig. 4) which is analogous to EDS analysis of AuNPs obtained from Aspergillus fumigatus [11].

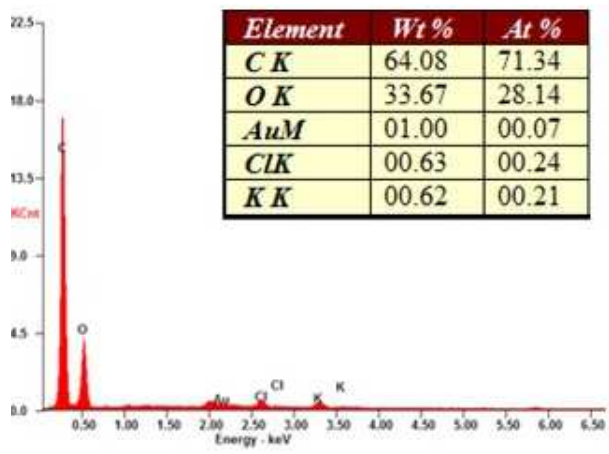

Fig. 4b: EDS analysis of biosynthesized AuNPs

\section{Zeta-potential}

Zeta potential is a key factor to evaluate the stability of nanoparticles dispersion. It was currently admitted the negative zeta potentials above $30 \mathrm{mV}$ required for maximum electrostatic stabilization. In our results, the negative zeta potential $-36.7 \mathrm{mV}$ in fig. 5 was observed using $2 \mathrm{mmol}$ aqueous gold solution with fungal biomass. The negative charge present in the core of nanoparticles matrixes is involved in electrostatic interaction with weakly basic drug molecules [28]. The presence of $\mathrm{OH}$ group on gold nanoparticles confirmed by the FTIR analysis.

\section{X-ray diffraction}

The synthesis and crystalline nature of the AuNPs were evaluated by Xray diffraction analysis, which shows the bioreduction and face-centered cubic structure of gold nanoparticles (fig. 6). Intense peaks observed at the angular positions at 2theta in the spectra (111), (200), (220) (311) and $(222)$ at $38.9^{\circ}, 43.4^{\circ}, 64.4^{\circ}, 78.7^{\circ}$ and $83.4^{\circ}$ agree to the Bragg's reflection of crystalline Gold nanoparticles. XRD analysis results were analogous to gold nanoparticles of Streptomyces hygroscopicus [29]. 

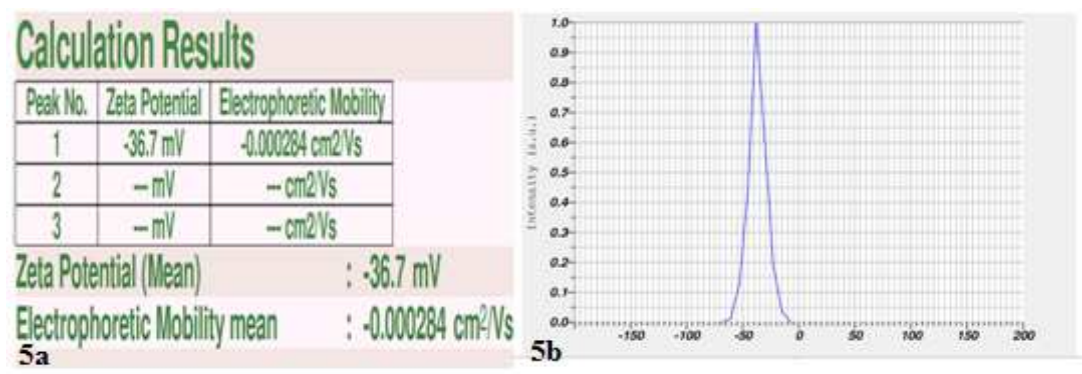

Fig. 5: Zeta potential of biosynthesized AuNPs

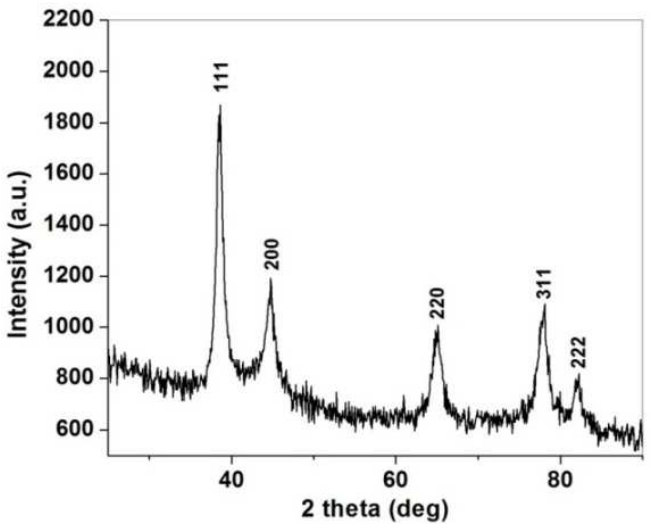

Fig. 6: XRD-pattern of biosynthesized AuNPs
Fourier transforms infrared spectroscopy analysis of gold nanoparticles

Different functional groups revealed with well know peaks in the infrared region of the electromagnetic spectrum shown in fig. 7. The absorption peak at $3436 \mathrm{~cm}^{-1}$ assigned as a polyphenolic $\mathrm{OH}$ group, $\mathrm{CH}$ stretching vibrations of alkanes cluster were observed at $2989 \mathrm{~cm}^{-1}$, a narrow band at 1644,2350 and $2562 \mathrm{~cm}^{-1}$ is represented as $\mathrm{C}=\mathrm{N}$ due to carbonyl stretch vibrations arise due to amide I and amide II functional groups present in protein secreted by the fungus act as linkage with AuNPs. The absorption peak at $1,455 \mathrm{~cm}^{-1}$ and $1,024 \mathrm{~cm}^{-1}$ assigned as the $\mathrm{C}-\mathrm{C}$ stretch vibration of an aromatic group and C-N stretching vibrations of aliphatic amines. These stretching vibrations are analogous to the vibrations of gold nanoparticles synthesized by Turbinaria conoides [30]. Finally, the narrow peak of alkyl halides revealed by weak bands in 738 and $599 \mathrm{~cm}^{-1}$. It indicates the enzymatic protein molecules present in Neosartorya udagawae act as stabilizing/binding agents and prevent its agglomeration

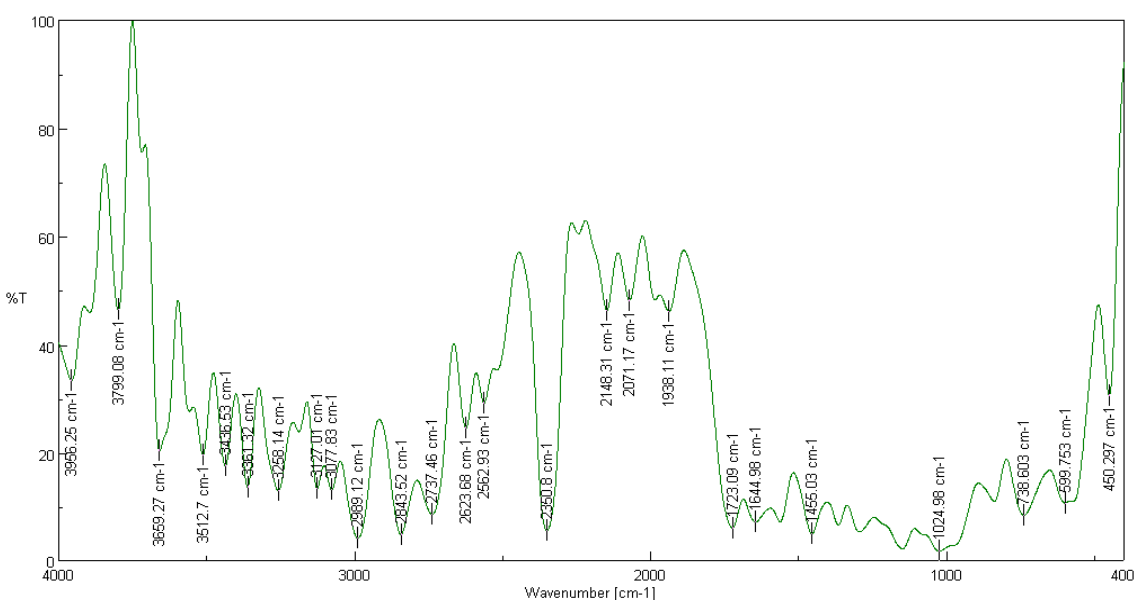

Fig. 7: FTIR spectra of biosynthesized AuNPs ranging from $1000-4000 \mathrm{~cm}^{-1}$

\section{Viability test}

The fungal growth when exposed to $2 \mathrm{mmol} \mathrm{HAuCl}_{4}$ display resistance by white cottony mycelium with a dull green appearance in the middle of the growth on CDA plates after $24 \mathrm{~h}$ of reaction (fig. 8). Untreated Neosartorya udagawae also showed good growth only in CDA supplemented with $2 \mathrm{mmol}$ concentration of $\mathrm{HAuCl}_{4}$. Thus, it was confirmed the tolerance of the organism Neosartorya udagawae to Gold metal and $\mathrm{HAuCl}_{4}$.

\section{In vitro stability of gold nanoparticles in various biological fluids}

The stability of nanoparticles in an aqueous environment over time is of concern in the biomedical application of nanoparticles [31]. Therefore, in vitro stability of fungal synthesized AuNPs was assessed in various biological fluids. The absorbance in aqueous mixtures changed immediately upon combining nanoparticle solution with BSA, $10 \% \mathrm{NaCl}$, PBS pH-5, PBS pH-7, PBS pH-9 solutions that mimic biological environments of the human body. The stability of the AuNPs assessed by UV-after $24 \mathrm{~h}$ incubation (fig.
9-13) had the plasmon resonance band in $\mathrm{BSA}, \mathrm{NaCl}, \mathrm{PBS}$ at different $\mathrm{pH}$ retained, and this confirmed the stability of the nanoparticles in all the media. This indicated that the AuNPs were intact and thereby demonstrates excellent in vitro stability in biological fluids, hence these gold nanoparticles can be used for therapy.

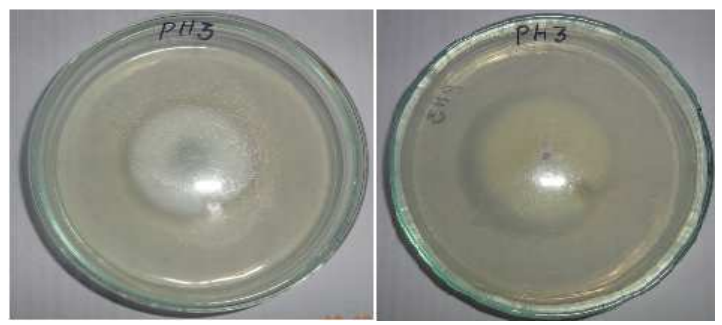

Fig. 8: Viability test of $N U$ on CDA after intracellular synthesis of AuNPs 


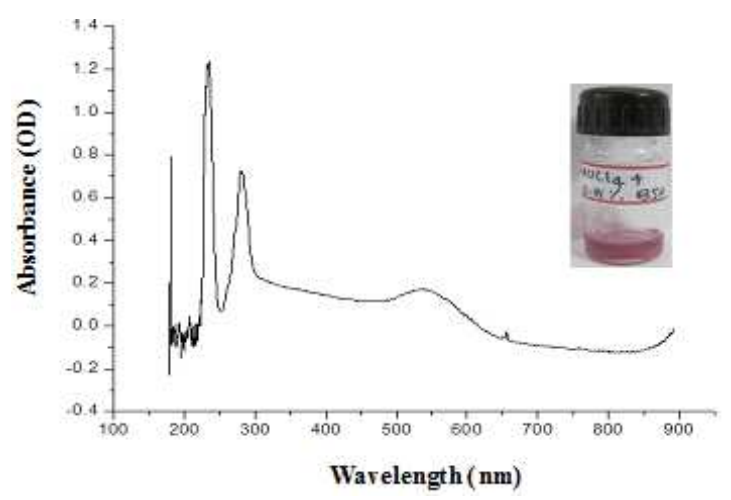

Fig. 9: The plasmon resonance band at $550 \mathrm{~nm}$ in BSA

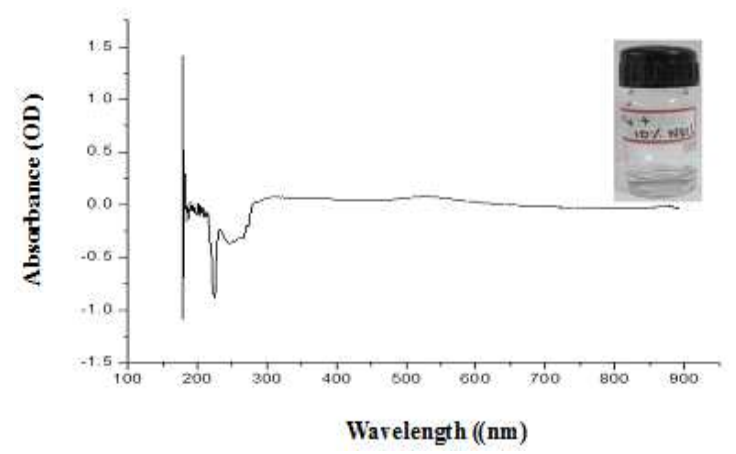

Fig. 10: The plasmon resonance band at $520 \mathrm{~nm}$ in $10 \%$ sodium chloride

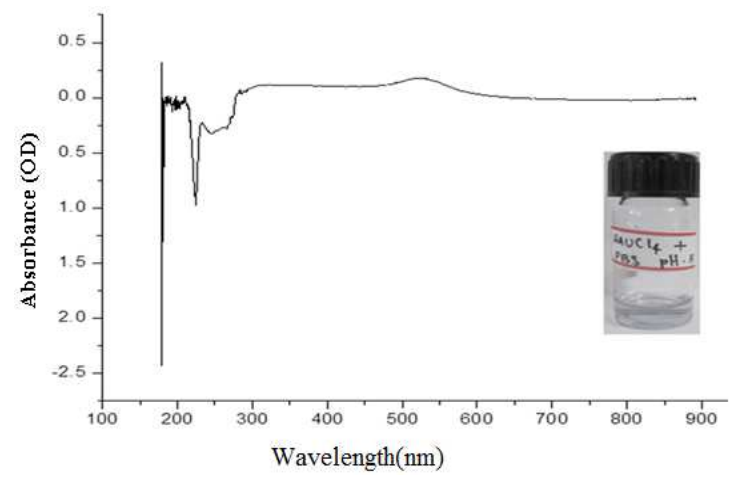

Fig. 11: The plasmon resonance band at $535 \mathrm{~nm}$ in Phosphate Buffer Saline at pH-5

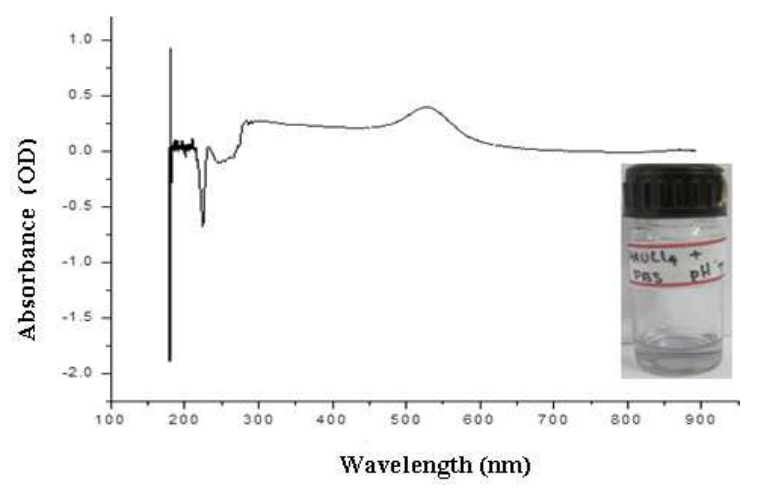

Fig. 12: The plasmon resonance band at $545 \mathrm{~nm}$ in phosphate buffer saline at $\mathrm{pH}-7$

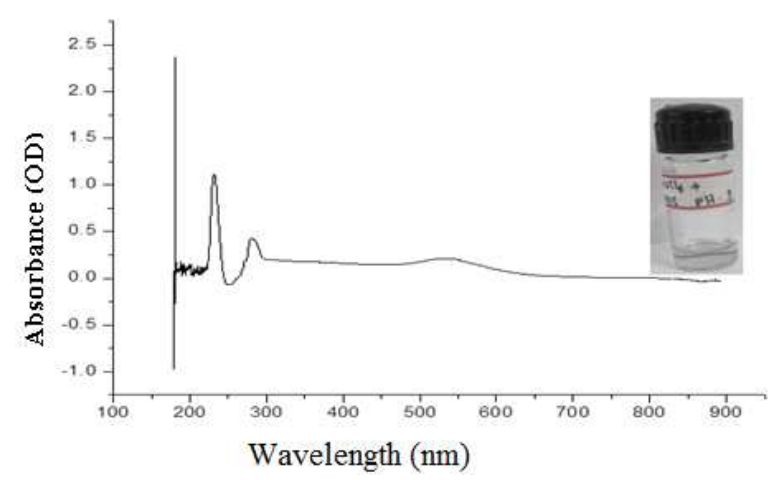

Fig. 13: The plasmon resonance band at $520 \mathrm{~nm}$ in phosphate buffer saline at $\mathrm{pH}-9$

\section{CONCLUSION}

Gold nanoparticles synthesized by Neosartorya udagawae were in the size of $50 \mathrm{~nm}$, TEM, and SEM analysis confirm the spherical and cubical shape of AuNPs. FTIR analyses specify the presence of functional groups $\mathrm{C}-\mathrm{N}, \mathrm{C}=\mathrm{N}, \mathrm{C}-\mathrm{C}, \mathrm{O}-\mathrm{H}$ and $\mathrm{C}-\mathrm{H}$ with distinguished peaks and these bonds offer stability by capping the AuNPs. The Bragg's reflections on XRD indicate the crystalline nature of nanoparticles synthesised and EDS confirm gold nanoparticles. The AuNPs were intact and thereby demonstrates excellent in vitro stability in biological fluids; Hence Neosartorya udagawae found to be a good candidate for the production of AuNPs and these AuNPs can be used for in vitro therapy.

\section{ACKNOWLEDGEMENT}

The authors are grateful to Prof. Dr. E. Somasundaram, Prof. Dr. N. Gunasekran and Dr. N. Natarajan of Tamil Nadu Agricultural University, Dr. K. Kadirvelu, DRDO of Bharathiyar University and special thanks to Dr. C. S. Shobana, G. R. D. College of Science, Coimbatore, Tamil Nadu, India for providing infrastructural support.

\section{CONFLICTS OF INTERESTS}

Declared none

\section{REFERENCES}

1. Jahn W. Review: chemical aspects of the use of gold clusters in structural biology. J Struct Biol 1999;127:106-12.

2. Peto G, Molnar GL, Paszti Z, Geszti O, Beck A, Guczi L. Electronic structure of gold nanoparticles deposited on Dihydroxy silicon phthalocyanine tetra sulfonic acid and oligo- $\mu$-oxo silicon phthalocyanine tetra sulfonic acid(siox/si). Mater Sci Eng C 2002;19:95-9.

3. Mona MA, Amira MM. Solid lipid nanoparticles and nanostructured lipid carriers of tolnaftate: design, optimization and in vitro evaluation. Int J Pharm Pharm Sci 2016;8:380-5.

4. Rashmi S, Preeti V, Sadhna P. Enzymatic formation of gold nanoparticles using Phanerochaete. Chrysosporium. Adv Chem Eng Sci 2011;1:154-62.

5. Meysam SN, Hosein SBG, Naimeh K. Biosynthesis of gold nanoparticles using Streptomyces Fulvissimus isolate. Nanomed J 2015;2:153-9.

6. Naveen BE, Prakash S. Biological synthesis of gold nanoparticles using marine algae Gracilaria corticata and its application as a potent antimicrobial and antioxidant agent. Asian J Pharm Clin Res 2013;6:179-82.

7. Mukherjee P, Ahmad A, Mandal D, Senapati S, Sainkar Sr, Khan $\mathrm{Mi}$, et al. Bioreduction of Aucl(-) ions by the fungus, Verticillium sp. and surface trapping of the gold nanoparticles. Angew Chem Int Ed Engl 2001;40:3585-8.

8. Saha K, Agasti SS, Kim C, Li X, Rotello VM. Gold nanoparticles in chemical and biological sensing. Chem Rev 2012;112:2739-79.

9. Zeinab S, Mojtaba S, Farzad K. Biological synthesis of gold nanoparticles by fungus Epicoccum nigrum. J Cluster Sci 2011;22:661-5. 
10. Absar A, Satyajyoti S, Islam MK, Rajiv K, Ramani R, Srinivas V, et al. Intracellular synthesis of gold nanoparticles by a novel alkali tolerant actinomycete, Rhodococcus Species. Nanotechnology 2003; 14:824-8.

11. Pranav Vasanthi B, Dilliganesh T, Vasanth KM, Chamundeeswari M, Baskar G. Biological synthesis and characterization of intracellular gold nanoparticles using biomass of Aspergillus fumigatus. Bull Mater Sci 2014;36:1201-5.

12. Balagurunathan $R$, Radhakrishnan $M$, Babu Rajendran $R$, Velmurugan D. Biosynthesis of gold nanoparticles by actinomycetes streptomyces viridogens strainhm10. Indian J Biochem Biophys 2011;48:331-5.

13. Jayshree N, Cynthia Pauline P, Kanchana A. Biogenic synthesis by Sphearanthus amaranthoids; towards the efficient production of the biocompatible gold nanoparticles'. Digest J Nanomater Biostructures 2012;7:123-33.

14. Xiangsheng Liu, Huang $\mathrm{N}$, Wang H, Huan Li, Jin Q, Jian Ji. The effect of ligand composition on the in vivo fate of multidenta poly (ethylene glycol) modified gold nanoparticles. Biomaterials 2013;34:8370-81.

15. Liangwei Du, Liang X, Jia-Xun F. Rapid extra-/intracellular biosynthesis of gold nanoparticles by the fungus Penicillium Sp. J Nanopart Res 2011;13:921-30.

16. Moghaddam KM. An introduction to the microbial metal nanoparticle preparation method. J Young Invest 2010;19:1-7.

17. Sneha K, Yeoung-Sang Y. Recovery of microbially synthesized gold nanoparticles using sodium citrate and detergents. Chem Eng J 2013;214:253-61.

18. Mandal D, Bolander ME, Mukhopadhyay D, Sarkar G, Mukherjee $P$. The use of microorganisms for the formation of metal nanoparticles and their application. Appl Microbiol Biotechnol 2006;69:485-92.

19. Narayanan KB, Sakthivel N. Biological synthesis of metal nanoparticles by microbes. Adv Colloid Interface Sci 2010;156:1-13.

20. Lengke M, Ravel B, Fleet ME, Wanger G, Gordon RA, Southam G. Mechanisms of gold bioaccumulation by filamentous cyanobacteria from gold (III)-chloride complex. Environ Sci Technol 2006b;40:6304-9.

21. Chakraborty N, Banerjee A, Lahiri S, Panda A, Ghosh AN, Pal R. Biorecovery of gold using cyanobacteria and eukaryotic alga with special reference to nanogold formation-a novel phenomenon. J Appl Phycol 2009;21:145-52.

22. Nayakm D, Nag M, Banerjee S, Pal R, Laskar S, Lahiri S Preconcentration of $198 \mathrm{Au}$ in a green alga, Rhizoclonium. J Radioanal Nucl Chem 2006;268:337-40.

23. Singaravelu G, Arockiamary JS, Ganesh Kumar V, Govindaraju K. A novel extracellular synthesis of monodisperse gold nanoparticles using marine alga, Sargassum wightii Greville. Colloids Surf B 2007;57:97-101.

24. Parial D, Patra HK, Dasgupta AK, Pal R. Screening of different algae for green synthesis of gold nanoparticle. Eur J Appl Physiol 2012b;47:22-9.

25. Dipannita P, Ruma P. Green synthesis of gold nanoparticles using cyanobacteria and their characterization. Indian J Appl Res 2014;4:69-72.

26. Sheikhlou Z, Salouti M, Farahmandkia Z, Mahmazi S, Einlou A. Intra-extra biosynthesis of gold nanoparticles by the fungus Rhizopus oryza' University of Zajan. University Med Sci 2012;20:47-56

27. Kalabegishvili T, Kirkesali Ei, Rcheulishvili An, Ginturi En, Murusidze Ig, Pataraya Dt, et al. Synthesis of gold nanoparticles by some strains of arthrobacter genera. J Mater Sci Eng 2012;2:164-73.

28. Vijayan V, Srinivasa Rao D, Jayachandra E, Anburaj J. Preparation and characterization of antidiabetic drug loaded solid lipid nanoparticles. J Innovative Trends Pharm Sci 2010;1:320-8.

29. Shivaji SW, Arvind MD, Zygmunt S. Biosynthesis, optimization, purification and characterization of gold nanoparticles. Afr J Microbiol Res 2014;8:138-46.

30. Shanmugam Rajeshkumar CM, Gnanadhas G, Kanniah P, Mahendran V, Chellapandian K, Gurusamy A. Seaweed-mediated synthesis of gold nanoparticles using Turbinaria conoides and its characterization. J Nanostruct Chem 2013;3:2-7.

31. Rohan SP, Anup H. Green synthesis of silver nanorods using an aqueous extract of kalanchoe pinnata fresh leaves and its synergistic effect with ciprofloxacin and antibiofilm activities. Int J Pharm Pharm Sci 2016;8:168-74.

\section{How to cite this article}

- V Jhansi Lakshmi, KP Kannan. Biosynthesis of gold nanoparticles by biosorption using Neosartorya udagawae: characterization and in vitro evaluation. Int J Pharm Pharm Sci 2016;8(11):108-113. 